\title{
Dificuldades dos médicos que atuam na Estratégia Saúde da Família de Minas Gerais para proverem atenção à saúde das mulheres
}

\author{
Difficulties of physicians working in the Family Health Strategy of Minas Gerais state to provide health care for women \\ Dificultades de los médicos que actúan en la Estrategia de Salud Familiar de Minas Gerais para proporcionar atención \\ a la salud de las mujeres
}

Victor Hugo Melo. Universidade Federal de Minas Gerais (UFMG). Belo Horizonte, MG, Brasil. victormelo@terra.com.br (Autor correspondente) Suzana Maria Pires do Rio. Faculdade de Medicina de Barbacena (FAME). Barbacena, MG, Brasil. suzanario@terra.com.br Rosuita Fratari Bonito. Universidade Federal de Uberlândia (UFU). Uberlândia, MG, Brasil. rosuita@netsite.com.br Claudia Teixeira Costa Lodi. Faculdade de Ciências Médicas de Minas Gerais (FCMMG). Belo Horizonte, MG, Brasil. claudiatclodi@gmail.com Maria Tereza Mohallem Fonseca. Universidade Federal de Minas Gerais (UFMG). Belo Horizonte, MG, Brasil. teresamohallem@terra.com.br Eliana Amaral. Universidade Estadual de Campinas (UNICAMP). Campinas, SP, Brasil. amaraleli@gmail.com

\section{Resumo}

Objetivo: Avaliar as dificuldades de médicos da Estratégia Saúde da Família (ESF) para proverem assistência à saúde das mulheres em todos os estágios de suas vidas. Métodos: Foi aplicado questionário semiestruturado a 203 médicos que atuam na ESF, em três regiões do Estado de Minas Gerais, com questões específicas relativas à atenção à saúde da mulher: prevenção do câncer do colo e das mamas, cuidado pré-natal e no puerpério, cuidados à saúde reprodutiva e atenção à saúde no climatério. Resultados: 0 motivo mais comum para serem médicos da ESF foi 0 interesse em trabalhar na Atenção Primária à Saúde $(38,4 \%)$. As dificuldades mais frequentes foram atender mulheres no climatério $(61,9 \%)$ e prover atenção à saúde reprodutiva $(41,9 \%)$. Comparando os médicos segundo o tempo de formação, verificou-se que os mais jovens (com até três anos de formação) apresentaram mais dificuldades em prover todos os tipos de cuidados à saúde das mulheres. A análise multivariada confirmou as limitações dos médicos mais jovens no atendimento ao climatério $(p<0,009)$ e a sua baixa participação em atividades educativas voltadas para as mulheres $(p<0,021)$. Conclusões: 0s médicos mais jovens apresentaram mais dificuldades específicas em todos os tipos de cuidados à saúde das mulheres e menor participação em atividades educativas. Presume-se que as dificuldades desses médicos estejam ligadas a deficiências na sua formação durante a graduação e à escassa participação nas ações programáticas de atenção à saúde das mulheres.

\section{Abstract}

Objective: To evaluate the difficulties of physicians from the Family Health Strategy (FHS) to provide health care for women in all stages of their lives. Methods: Two hundred and three physicians from FHS in three regions of the state of Minas Gerais, Brazil answered a semi-structured questionnaire with specific questions regarding women's healthcare, such as cervical and breast cancer prevention, prenatal and postpartum care, reproductive health care, and health care during climacteric. Results: The most common reason for having become doctors at the FHS program was the interest to work in Primary Health Care (38.4\%). The most frequent difficulties were taking care of climacteric women (61.9\%) and providing reproductive health care (41.9\%). By comparing physicians according to their practicing time since graduation, it was possible to find that the youngest (up to three years after graduation) presented more difficulty in providing all types of health care for women. Multivariate analysis confirmed the limitations of young physicians in taking care of perimenopausal women $(p<0.009)$ and their small participation in educational activities for women $(p<0.021)$. Conclusions: Younger doctors present more specific problems in all types of health care for women, with less participation in educational activities. It is assumed that these physicians' difficulties are linked to flaws during their undergraduate education and to a reduced participation in programmatic actions in women's health care.
Palavras-chave:

Saúde da Família

Competência Clínica

Medicina de Família e Comunidade Atenção Primária à Saúde

Educação Médica

\section{Keywords:}

Family Health Clinical Competence Family Practice Primary Health Care Education, Medical
Fonte de financiamento: declaram não haver. Parecer CEP:

UFMG parecer ETIC 0457.0.203.000-10. 


\section{Resumen}

Objetivo: Evaluar las dificultades de los médicos de la Estrategia de Salud Familiar (ESF), para proporcionar atención a la salud de las mujeres en todas las etapas de sus vidas. Métodos: Se aplicó un cuestionario semiestructurado a 203 médicos que trabajan en la ESF, pertenecientes a tres regiones del estado de Minas Gerais, con preguntas específicas relacionadas con la atención a la salud de la mujer: prevención del cáncer cérvico-uterino y de mama; atención prenatal y puerperio; cuidados de la salud reproductiva y atención de la salud durante el climaterio. Resultados: La razón más común para convertirse en médicos de la ESF fue su interés en trabajar con la Atención Primaria de Salud (38,4\%). Las dificultades más frecuentes fueron la de ayudar a las mujeres en el climaterio (61,9\%) y la de proporcionar servicios de salud reproductiva (41,9\%). Al comparar los médicos, en función del tiempo trascurrido tras su graduación, se verificó que las personas más jóvenes (hasta tres años tras su graduación) presentaron más dificultades al prestar los diferentes tipos de cuidados en la atención de la salud para las mujeres. El análisis multivariado confirmó las limitaciones de médicos jóvenes en el cuidado del climaterio $(p<0,009)$ y su baja participación en las actividades educativas dirigidas a las mujeres $(p<0,021)$. Conclusiones: Los médicos más jóvenes presentaron problemas más específicos en todos los tipos de atención de la salud de las mujeres y tuvieron una menor participación en las actividades educativas. Se supone que las dificultades de estos médicos estén relacionadas con deficiencias en su formación durante la graduación y con su limitada participación en las actividades del programa de la atención de la salud de las mujeres.
Palabras clave:

Salud de la Familia

Competencia Clínica

Medicina Familiar y Comunitaria Atención Primaria de Salud Educación Médica

\section{Introdução}

A Medicina de Família e Comunidade (MFC), cujos princípios conceituais foram introduzidos no Brasil na década de 1970, surgiu como um movimento não somente para responder aos altos custos e à crescente especialização da assistência médica como também para resgatar a promoção e proteção da saúde dos indivíduos. Até então, a assistência médica individual e curativa ficava a cargo da Previdência Social, enquanto os cuidados preventivos e coletivos eram da responsabilidade do Ministério da Saúde. ${ }^{1,2}$ A Conferência de Alma Ata, realizada em 1978, estimulou o surgimento de várias experiências isoladas em países desenvolvidos e em desenvolvimento, inclusive no Brasil, com o intuito de prover cuidados primários de saúde como direito fundamental. ${ }^{3,4}$

Por sua vez, a baixa resolubilidade do modelo de assistência à saúde e a exclusão social de parte da população contribuíram para que fossem definidos na Constituição de 1988 três principais referenciais do sistema de saúde brasileiro: conceito ampliado de saúde, saúde como direito do cidadão e dever do Estado e a criação de um Sistema Único de Saúde (SUS) fundamentado nos princípios da universalidade, integralidade, descentralização e participação comunitária. ${ }^{5}$

A implantação do Programa Saúde da Família (PSF) em 1994, visou à reorientação da Atenção Primária à Saúde (APS) como porta de entrada aos serviços de saúde para as populaçóes desfavorecidas, associando açóes de promoçáo da saúde/ prevenção de agravos nos diferentes níveis de atenção. ${ }^{6}$ Em 1995, o PSF passou a fazer parte da política oficial do governo do Estado de Minas Gerais. ${ }^{7}$ Atualmente, 820 municípios do estado (97,5\%) tem pelo menos uma equipe de saúde da família. Dados do governo de Minas mostram que em abril de 2012 havia 4.319 equipes em atividade, representando uma cobertura de $76 \%$ da populaçáo mineira. ${ }^{8}$

O PSF foi transmutado em Estratégia Saúde da Família (ESF) em 1998, buscando romper o paradigma da atenção simplificada/baixo custo para a prestação de cuidados resolutivos, ${ }^{6}$ fundamental para o fortalecimento e atuação da MFC. A ESF, enquanto estratégia para reorganização da APS, é porta de entrada prioritária do SUS, tendo como principais características: equidade, integralidade, continuidade da atenção, coordenação da assistência dentro do próprio sistema e participação comunitária. ${ }^{9}$

Entre as diversas áreas de atuação da ESF uma das prioridades é a atenção integral à saúde da mulher, englobando estratégias que contemplem os objetivos do Programa de Assistência Integral à Saúde da Mulher (PAISM) de forma interdisciplinar e no formato de apoio matricial. Como aspectos fundamentais do PAISM destacam-se: atenção qualificada às mulheres com queixas ginecológicas em todas as fases dos ciclos de vida - infância, adolescência e fase adulta (menacme, climatério e menopausa); atenção às doenças infecciosas (DSTs), atenção à saúde sexual/reprodutiva (planejamento familiar, infertilidade, anticoncepção), pré-natal qualificado, além da assistência ao puerpério; açôes de saúde mental; atenção à violência contra a mulher; atenção às principais doenças crônicas e degenerativas (hipertensão, diabetes, cânceres de mama e colo uterino); inclusão da visão de gênero, raça e etnias. ${ }^{9}$

A consolidação da ESF tem apresentado inúmeras dificuldades operacionais para sua implantação e manutenção e um dos principais problemas é a deficiente fixação dos médicos, ${ }^{1,10,11}$ sendo observada elevada rotatividade entre estes profissionais. ${ }^{11-13}$ Vários fatores são apontados como causas desse problema, dentre os quais: contratação em período integral de trabalho; desvalorização profissional pelos próprios colegas; falta de entendimento da população sobre a ESF; e melhores possibilidades salariais em outras atividades. ${ }^{1,11}$ Outro aspecto é o fato de muitos médicos ingressarem na ESF sem o perfil adequado para 
atuar na saúde comunitária. ${ }^{12-14}$ Estudos apontam também falhas no sistema de referência e contrarreferência como fatores que dificultam a permanência dos médicos. ${ }^{1,6,10,11}$ De maneira constante e uníssona, autores chamam a atenção para a falta de capacitação destes profissionais como outro obstáculo para a sua fixação na ESF, o que aumenta, consequentemente, a sua rotatividade. ${ }^{1,6,11-13,15}$

Desde que foram instituídos, o PSF e a ESF experimentaram crescimento exponencial e, entre seus maiores êxitos, está a extensão do cuidado à saúde para grande parcela da população pobre brasileira. Entretanto, a rapidez de sua implantação acarretou a incorporação de pessoas com formação ou perfil nem sempre adequados para compor as equipes, o que tem comprometido a legitimidade da proposta e sua capacidade para substituir modelos tradicionais. ${ }^{16}$

A proposta da ESF é que o médico deve ter competências preventivas, terapêuticas e de gestão de recursos, além de solucionar os problemas de saúde indiferenciados que lhe são apresentados. Na prática, observa-se grande número de profissionais com baixa qualificação e inúmeras atribuiçôes. ${ }^{13}$ Esse fato evidencia a necessidade de se promover a revisão dos currículos de graduação da área médica, enfatizando a responsabilidade social da formação, além da importância da educação permanente desses profissionais.

O Programa Nacional de Reorientação da Formação Profissional em Saúde (Pró-Saúde) foi criado em 2005 com o objetivo de promover integração ensino-serviço e reorientação da formação profissional - com ênfase na APS e na abordagem integral dos indivíduos. ${ }^{17} \mathrm{~A}$ articulação entre ESF e Pró-Saúde pode fomentar a parceria entre as Instituiçóes de Ensino Superior e os Serviços Públicos de Saúde, contribuindo para a potencialização das respostas às necessidades de saúde da população brasileira, mediante formação adequada de recursos humanos e produção do conhecimento voltada para a prestaçáo de serviços.

O objetivo do presente estudo foi avaliar as dificuldades dos médicos que atuam na ESF do Estado de Minas Gerais para prestar cuidados à saúde das mulheres, de forma a discutir junto às instancias de formação, as habilidades e conhecimentos que necessitam desenvolver, durante e após o curso médico, para proverem atenção com qualidade.

\section{Métodos}

O estudo foi desenvolvido no Estado de Minas Gerais, no período de maio de 2010 a março de 2011. Foram convidados a participar 203 médicos inscritos no Programa de Educação Permanente para Médicos de Família (PEP) ${ }^{18}$ da Secretaria de Estado da Saúde (SES) de Minas Gerais, em diferentes regiôes do estado. Os médicos responderam a um questionário semiestruturado contendo questóes gerais sobre sua formação/capacitação prévia para atuarem como médicos da ESF. A parte de respostas específicas apresentava questóes relacionadas aos cuidados de atenção à saúde das mulheres, previstas na ESF: prevenção dos cânceres de colo uterino e das mamas; atenção pré-natal e no puerpério; atenção à saúde reprodutiva e atenção no climatério. ${ }^{9,19,20} \mathrm{O}$ questionário continha questôes fechadas (estruturadas) nas quais os médicos respondiam se sabiam ou năo realizar procedimentos, solicitar exames, prescrever ou identificar situaçóes de risco. Depois de responder a uma questão fechada, o médico respondia livremente a uma questão aberta (semiestruturada) relacionada à resposta estruturada anterior. Os questionários foram respondidos pelos médicos em reuniôes estratégicas educacionais do PEP, após assinarem Termo de Consentimento Livre e Esclarecido (TCLE).

Para análise dos dados, os médicos foram agrupados de acordo com o tempo desde o término da graduaçáo (tempo de formação), tendo sido escolhido o período de três anos como ponto de corte, levando-se em consideração, principalmente, que prestar cuidados de atenção à saúde das mulheres é parte do aprendizado dos alunos em qualquer curso de medicina. Ao mesmo tempo, caso não tivessem segurança para realizar essa atenção após sua graduação, neste período de até três anos, poderiam ter recebido treinamento para prover tais cuidados.

A análise estatística foi dividida em três etapas: a) caracterização da amostra, realizando-se análise descritiva e distribuição das frequências das características gerais dos médicos, das atividades desenvolvidas na ESF e das dificuldades específicas mais comuns no cuidado à saúde das mulheres; b) análise univariada, comparando os anos de formação com todas as variáveis categóricas envolvidas. Para esta análise, foi utilizado o teste do quiquadrado, considerando-se significantes os resultados com p<0,05 e c) análise multivariada, utilizando-se a regressão binária logística para avaliar quais variáveis selecionadas tinham associação independente com o tempo de formação. Foram selecionadas variáveis que apresentavam valor p $<0,20$ na análise univariada e considerados significantes resultados com $\mathrm{p}<0,05$. Para ajuste final no modelo multivariado, foram excluídos 18 médicos com residência e/ou especialização em Ginecologia e Obstetrícia. Os dados foram digitados, armazenados e analisados no software Statistical Package for the Social Sciences (SPSS), versão 12.0. O estudo foi aprovado pelo Comitê de Ética em Pesquisa da Universidade Federal de Minas Gerais (Parecer ETIC 0457.0.203.000-10). 


\section{Resultados}

Os questionários foram respondidos por 203 médicos da ESF do PEP/SES/MG em três diferentes regióes do Estado de Minas Gerais: regiáo metropolitana de Belo Horizonte $(n=156)$; Triângulo Norte $(n=28)$; Vertentes $(n=19)$. Todos os médicos solicitados responderam ao questionário proposto, não havendo, portanto, recusas ou perdas.

A Tabela 1 apresenta características gerais dos médicos entrevistados, na qual se observa que 55,0\% formaram-se em escolas públicas e 62,6\% tinham mais de três anos de formado. Com relação à especialização/residência médica (RM), observou-se que, entre os médicos que realizaram RM, quase todos informaram especialização na mesma área de atuação, sendo que alguns médicos informaram terem realizado mais de um tipo de especialização. Entre os 203 médicos, 121 (59,6\%) haviam realizado alguma especialização e, entre os 82 médicos que não fizeram nenhuma especialização, 64 tinham menos de três anos de formação. As especializaçôes mais frequentes foram Medicina de Família e Comunidade (24,1\%); Medicina do Trabalho (10,3\%); Ginecologia e Obstetrícia $(8,9 \%)$, porém somente 68 médicos fizeram alguma residência médica. Entre os 135 médicos que não fizeram nenhuma residência médica, 70 tinham menos de três anos de formação. As residências médicas mais frequentes foram: Ginecologia e Obstetrícia (8,9\%), Pediatria (6,4\%) e Medicina de Família e Comunidade $(5,9 \%)$. Convém enfatizar que os médicos que fizeram residência médica em GO foram os mesmos que realizaram especialização

Tabela 1. Características gerais dos médicos participantes da pesquisa.

\begin{tabular}{|c|c|c|}
\hline Características & $\mathrm{N}=203$ & n $\%$ \\
\hline \multicolumn{3}{|c|}{ Tipo de Faculdade de Medicina em que se formou ${ }^{a}$} \\
\hline Pública & 110 & 55,0 \\
\hline Privada & 87 & 43,5 \\
\hline Fundações & 3 & 1,5 \\
\hline \multicolumn{3}{|l|}{ Tempo de formado } \\
\hline Mais de três anos & 127 & 62,6 \\
\hline Até três anos & 76 & 37,4 \\
\hline Fez especialização & 121 & 59,6 \\
\hline Medicina de Família e Comunidade & 49 & 24,1 \\
\hline Medicina do Trabalho & 21 & 10,3 \\
\hline Ginecologia e Obstetrícia & 18 & 8,9 \\
\hline Outras especializações & 33 & 16,2 \\
\hline Fez residência médica & 68 & 33,5 \\
\hline Ginecologia e Obstetrícia & 18 & 8,9 \\
\hline Pediatria & 13 & 6,4 \\
\hline Medicina de Família e Comunidade & 12 & 5,9 \\
\hline Outras residências médicas & 25 & 12,3 \\
\hline \multicolumn{3}{|c|}{ Experiência anterior no cuidado de mulheres ${ }^{b}$} \\
\hline $\operatorname{Sim}$ & 99 & 48,8 \\
\hline Durante a graduação & 42 & 20,7 \\
\hline Não & 88 & 43,3 \\
\hline \multicolumn{3}{|l|}{ Por que ser médico do PSFc } \\
\hline Gosta de trabalhar com atenção básica & 78 & 38,4 \\
\hline Salário/Emprego/Horário de trabalho & 63 & 31,0 \\
\hline Gosta de trabalhar com a comunidade & 25 & 12,3 \\
\hline Outros motivos & 29 & 18,3 \\
\hline \multicolumn{3}{|l|}{ População das cidades (mil habitantes) } \\
\hline$<50$ & 62 & 30,5 \\
\hline Entre 50 e 100 & 36 & 17,8 \\
\hline Entre 100 e 200 & 38 & 18,7 \\
\hline$>200$ & 67 & 33,0 \\
\hline \multicolumn{3}{|c|}{ Tempo de atuação no PSF (anos completos) } \\
\hline$\geq 1$ ano (variação 1 - 16 anos) & 152 & 74,9 \\
\hline < 1 ano (variação 1 - 11 meses) & 51 & 25,1 \\
\hline Outras características & & Variação \\
\hline Mediana da idade (anos) & 35 & $23-80$ \\
\hline
\end{tabular}


nessa área de atuação. Somente 99 (48,8\%) médicos relataram experiência prévia no cuidado à saúde das mulheres e, entre eles, $42(20,7 \%)$ responderam ter realizado estas atividades durante a graduação. Quanto aos motivos que os levaram a se tornarem médicos da ESF, os mais frequentes foram: gostar de atuar na APS (38,4\%); questôes relacionadas a emprego, salário e horário de trabalho (31,0\%); e gostar de trabalhar com a comunidade (12,3\%). Nessa amostra, $152(74,9 \%)$ médicos atuavam na ESF há mais de um ano.

A Tabela 2 apresenta atividades desenvolvidas pelos médicos da ESF no cuidado à saúde das mulheres, estratificadas em diferentes tipos de cuidados. Pode-se perceber que 85,2\% informaram saber realizar exame ginecológico para prevenção do câncer cervical, 47,8\% faziam coleta de amostras para exame de Papanicolaou. Papanicolaou e somente 27,4\% relataram alguma dificuldade específica na realização desse exame. No que concerne ao exame das mamas para prevenção do câncer, $98,5 \%$ informaram saber realizá-lo, mas somente $59,6 \%$ o faziam.

Tabela 2. Atividades desenvolvidas pelos médicos e suas dificuldades específicas no cuidado à saúde das mulheres.

\begin{tabular}{|c|c|c|}
\hline Atividades & $\mathrm{n} / \mathrm{N}^{\mathrm{a}}$ & $\mathrm{n} \%$ \\
\hline \multicolumn{3}{|l|}{ Exame Ginecológico (prevenção do câncer cervical) } \\
\hline Sabe realizar o exame & $173 / 203$ & 85,2 \\
\hline Identifica a JEC & $184 / 203$ & 90,6 \\
\hline Faz coleta de Papanicolaou & 97/203 & 47,8 \\
\hline Usa espátula + escova & $124 / 201$ & 61,7 \\
\hline Faz teste de Schiller & $101 / 203$ & 49,8 \\
\hline Interpreta Schiller & $185 / 201$ & 92,0 \\
\hline Dificuldades (sim) & 49/179 & 27,4 \\
\hline \multicolumn{3}{|l|}{ Exame das Mamas (prevenção do câncer de mama) } \\
\hline Sabe realizar o exame & $200 / 203$ & 98,5 \\
\hline Realiza exame das mamas & $121 / 203$ & 59,6 \\
\hline Dificuldades (sim) & $30 / 191$ & 15,7 \\
\hline Solicita mamografia & $122 / 201$ & 60,7 \\
\hline \multicolumn{3}{|l|}{ Atenção Pré-natal } \\
\hline Atende gestantes & $177 / 202$ & 87,6 \\
\hline Solicita exames complementares & $179 / 203$ & 88,1 \\
\hline Prescreve vacina antitetânica & $143 / 203$ & 70,4 \\
\hline Indica vacina para Hepatite B & 108/182 & 59,3 \\
\hline Participa de atividades educativas & 98/199 & 49,2 \\
\hline \multicolumn{3}{|l|}{ Realiza toque vaginal para avaliar: } \\
\hline Colo uterino & $164 / 202$ & 81,2 \\
\hline Apresentação fetal & $134 / 202$ & 66,3 \\
\hline Bacia materna & 87/199 & 43,7 \\
\hline Dificuldades no cuidado pré-natal (sim) & $43 / 195$ & 22,1 \\
\hline \multicolumn{3}{|l|}{ Atenção no Puerpério } \\
\hline Atende puérperas & $155 / 203$ & 76,4 \\
\hline Identifica os fatores de risco & $100 / 203$ & 49,2 \\
\hline Dificuldades (sim) & $67 / 194$ & 34,5 \\
\hline \multicolumn{3}{|l|}{ Atenção no Climatério } \\
\hline Atende mulheres climatéricas & $140 / 202$ & 69,3 \\
\hline Rastreia ou investiga doenças & $57 / 161$ & 35,4 \\
\hline Solicita exames complementares & $41 / 149$ & 27,5 \\
\hline Prescreve hormônios & $27 / 124$ & 21,8 \\
\hline Participa de atividades educativas & $56 / 203$ & 27,6 \\
\hline Dificuldades (sim) & $122 / 197$ & 61,9 \\
\hline \multicolumn{3}{|l|}{ Atenção à Saúde Reprodutiva } \\
\hline Atende estas mulheres & $152 / 201$ & 75,6 \\
\hline Conhece os métodos contraceptivos fornecidos pelo SUS & 135/193 & 69,9 \\
\hline Prescreve contracepção de emergência & $119 / 160$ & 74,3 \\
\hline Solicita exames complementares & $17 / 115$ & 14,8 \\
\hline Conhece a lei 9263/96 & $115 / 200$ & 57,5 \\
\hline Participa de atividades educativas & $84 / 201$ & 41,8 \\
\hline Dificuldades (sim) & $83 / 198$ & 41,9 \\
\hline
\end{tabular}

${ }^{a}$ Foram excluídos os médicos que não responderam à pergunta, em cada variável. JEC - Junção escamocolunar. 
Ainda na Tabela 2, são apresentadas atividades de atenção pré-natal, podendo-se perceber que 87,6\% dos médicos atendiam gestantes e $88,1 \%$ solicitavam exames rotineiros. Com relação às atividades educativas voltadas para as mulheres, quase metade dos médicos $(49,2 \%)$ tinha alguma participação. Percebe-se que $81,2 \%$ avaliavam o colo uterino, ao toque bimanual e $66,3 \%$ identificavam o polo fetal que se apresenta na pelve. Somente $22,1 \%$ relataram dificuldades para atender as gestantes. Depois do parto, 76,4\% atendiam as puérperas, mas somente $49,2 \%$ identificavam fatores de risco puerperais. Um terço $(34,5 \%)$ dos médicos relatou alguma dificuldade para atender essas pacientes.

A Tabela 2 também apresenta informaçôes sobre atividades com mulheres climatéricas e atenção à saúde reprodutiva. Apesar de dois terços $(69,3 \%)$ informarem que atendiam mulheres no climatério, 61,9\% relataram ter dificuldades para prestar cuidados à saúde dessas mulheres. Por sua vez, 75,6\% prestavam cuidados à saúde reprodutiva e $69,9 \%$ conheciam os métodos contraceptivos disponíveis no SUS. Por fim, 41,9\% relataram dificuldades para prestar cuidados à saúde reprodutiva.

A Tabela 3 apresenta um resumo das dificuldades específicas mais comuns relatadas pelos médicos no atendimento às mulheres.

Tabela 3. Dificuldades específicas mais frequentes dos médicos no atendimento às mulheres.

\begin{tabular}{lcc}
\hline \multicolumn{1}{c}{ Dificuldades } & $\mathrm{n} / \mathrm{N}^{\mathrm{a}}$ & $\mathrm{n} \%$ \\
\hline Exame Ginecológico (prevenção do câncer cervical) & $49 / 179$ & 27,4 \\
Exame das Mamas (prevenção do câncer de mama) & $30 / 191$ & 15,7 \\
Atenção Pré-natal & $43 / 195$ & 22,1 \\
Atenção no Puerpério & $67 / 194$ & 34,5 \\
Atenção no Climatério & $122 / 197$ & 61,9 \\
Atenção à Saúde Reprodutiva & $83 / 198$ & 41,9 \\
\hline
\end{tabular}

a Foram excluídos os médicos que não responderam à pergunta, em cada variável.

$\mathrm{Na}$ Tabela 4, são apresentadas todas as variáveis que mostraram diferenças significantes entre os médicos, segundo o tempo de formação, na análise univariada.

Tabela 4. Análise univariada comparando anos de formação e outras variáveisa ${ }^{a}$

\begin{tabular}{|c|c|c|c|c|}
\hline Variáveis & $\begin{array}{l}\leq 3 \text { anos } \\
n / 76(\%)\end{array}$ & $\begin{array}{c}>3 \text { anos } \\
n / 127(\%)\end{array}$ & $\begin{array}{c}\text { Total } \\
\text { n/203 (\%) }\end{array}$ & Valor $p$ \\
\hline \multicolumn{5}{|c|}{ Exame Ginecológico (prevenção do câncer cervical) } \\
\hline Faz coleta de Papanicolaou (não) & $52(68,4)$ & $73(42,5)$ & $106(52,2)$ & 0,000 \\
\hline Usa espátula e escovinha (não) & $34(44,7)$ & $43(33,9)$ & $77(37,9)$ & 0,04 \\
\hline Identifica o que é JEC (não) & $3(3,9)$ & $16(12,6)$ & $19(9,4)$ & 0,041 \\
\hline \multicolumn{5}{|c|}{ Exame das Mamas (prevenção do câncer de mama) } \\
\hline Examina as mamas (não) & $3(3,9)$ & $0(0,0)$ & $3(1,5)$ & 0,024 \\
\hline \multicolumn{5}{|l|}{ Atenção Pré-natal } \\
\hline Atividade Educativa (não) & $47(61,8)$ & $54(42,5)$ & $101(49,8)$ & 0,014 \\
\hline \multicolumn{5}{|l|}{ Atenção no Puerpério } \\
\hline Atende puérperas (não) & $24(31,6)$ & $24(18,9)$ & $48(23,6)$ & 0,04 \\
\hline Dificuldades (sim) & $41(53,9)$ & $26(20,5)$ & $67(33,0)$ & 0,000 \\
\hline \multicolumn{5}{|l|}{ Atenção no Climatério } \\
\hline Rastreia ou investiga doenças (não) & $37(48,7)$ & $31(24,4)$ & $68(33,5)$ & 0.000 \\
\hline Solicita exames (não) & $36(47,4)$ & $35(27,6)$ & $71(35,0)$ & 0,000 \\
\hline Prescreve hormônios (não) & $54(71,1)$ & $65(51,2)$ & $119(58,6)$ & 0,007 \\
\hline Atividade educativa (não) & $66(86,8)$ & $81(63,8)$ & $147(72,4)$ & 0,000 \\
\hline Dificuldades (sim) & $65(85,5)$ & $57(44,9)$ & $122(60,1)$ & 0,000 \\
\hline \multicolumn{5}{|l|}{ Atenção à Saúde Reprodutiva } \\
\hline Solicita exames (não) & $37(48,7)$ & $37(29,1)$ & $74(36,5)$ & 0,015 \\
\hline Atividade Educativa (não) & $53(69,7)$ & $64(50,4)$ & $117(57,6)$ & 0,019 \\
\hline Dificuldades (sim) & $16(19,3)$ & $67(52,8)$ & $83(40,9)$ & 0,000 \\
\hline
\end{tabular}

a Foram listadas somente as variáveis com diferença significante. JEC - Junção escamocolunar. 
Os resultados do modelo final da análise multivariada estấo apresentados na Tabela 5. Todas as variáveis apresentadas na Tabela 4 entraram na análise final de regressão binária logística. Para esta análise, foram excluídos 18 médicos com residência/ especialização em Ginecologia e Obstetrícia. Como evidenciado pelos valores de razóes de chances (OR Ajustados), os médicos com até três anos de formação faziam menos coleta de amostras para exame de Papanicolaou $(\mathrm{OR}=2,31)$ - embora identificassem com mais facilidade a junção escamocolunar (JEC) $(\mathrm{OR}=0,14)$. Ao mesmo tempo, rastreavam menos as doenças no climatério $(\mathrm{OR}=0,57)$ e tinham menor participação em atividades educativas nesse período de vida das mulheres $(\mathrm{OR}=2,65)$, quando comparados aos médicos com mais de três anos de formação.

Tabela 5. Modelo final da análise multivariadaa .

\begin{tabular}{lccc}
\multicolumn{1}{c}{ Médicos com até três anos de formação } & Valor p & OR Ajustado & IC a 95\% \\
\hline Não identificam a JEC & 0,004 & 0,14 & $0,036-0,528$ \\
Não fazem coleta de Papanicolaou & 0,015 & 2,31 & $1,179-4,526$ \\
Rastreiam doenças do climatério & 0,009 & 0,57 & $0,373-0,868$ \\
Não participam de atividades educativas no climatério & 0,021 & 2,65 & $1,162-6,052$ \\
\hline a Excluídos 18 médicos com residência/especialização em Ginecologia e Obstetrícia. JEC - Junção escamocolunar. OR \\
Ajustado - Odds Ratio Ajustado.
\end{tabular}

\section{Discussão}

A literatura sobre o tema é escassa, não tendo sido encontrada publicação específica para comparar com os resultados deste estudo, e a amostra foi considerada representativa dos médicos da ESF do Estado de Minas Gerais. Outro aspecto favorável foi a diversidade de escolas médicas - tanto públicas quanto privadas, inclusive de outros estados da federação - em que os médicos tiveram sua formação.

Os médicos foram divididos em dois grupos de acordo com o tempo de formação, pois, além de detectar as dificuldades apresentadas no atendimento das mulheres, buscava-se associá-las com prováveis deficiências de formação na graduação. Estabeleceu-se o período de três anos de formação para criar os subgrupos, o que correspondeu a 76 médicos entrevistados (37,4\% da amostra). Este percentual foi semelhante ao encontrado em publicaçáo recente (39,0\%), na qual os autores utilizaram um ponto de corte de até cinco anos de formação. ${ }^{1}$ Considerou-se que o ponto de corte em até três anos de formado foi adequado para avaliar possíveis lacunas de formação durante a graduação, que poderiam ser sanadas caso cursassem residência ou alguma especializaçáo. Destaca-se que 68 médicos $(33,5 \%)$ de toda a amostra fizeram residência médica e que Ginecologia e Obstetrícia esteve entre as mais frequentes ( $\mathrm{n}=18 ; 8,9 \%)$.

O principal motivo para se tornar médico da ESF foi gostar de atuar na APS (38,4\%). Este percentual foi maior quando comparado ao estudo de Vasconcelos e Zaniboni ${ }^{1}$ que encontrou percentual de $25 \%$.

As Diretrizes Curriculares Nacionais (DCN) do Curso de Graduação em Medicina, instituídas pelo Conselho Nacional de Educação em 2001, estabeleceram as prerrogativas que devem ser seguidas pelas instituiçóes do Sistema de Educação Superior do País, tendo como base conhecimentos gerais e compromisso social..$^{21}$ Entre as prerrogativas contidas nas DCN, destacam-se: "O Curso de graduação em Medicina tem como perfil... o médico, com formação generalista, humanista..." com capacidade de atuar "... em seus diferentes nivveis de atenção, com açóes de promoção, prevenção, recuperação e reabilitação à saúde, na perspectiva da integralidade da assistência”... Por outro lado, O estágio curricular obrigatório de treinamento em serviço incluirá necessariamente aspectos essenciais nas áreas de Clínica Médica, Cirurgia, Ginecologia-Obstetrícia, Pediatria e Saúde Coletiva, devendo incluir atividades no primeiro, segundo e terceiro níveis de atenção em cada área. ${ }^{22}$

Fica claro que a atenção à saúde da mulher deve fazer parte do conteúdo curricular das escolas médicas e, por isso, é preocupante o fato de apenas $20,7 \%$ dos médicos relatarem ter participado da atenção à saúde das mulheres durante a graduação. Temos três hipóteses para interpretar esse resultado: a) os médicos não entenderam a pergunta; b) as escolas médicas não oferecem aos seus alunos campo de prática específico para que possam prestar estes cuidados; c) os médicos, quando estudantes, podem não ter se envolvido com os cuidados à saúde das mulheres porque não gostavam, ou porque estavam interessados em outra especialidade, ou ainda, porque não foram suficientemente motivados por seus professores. 
Apesar da pouca participação em cuidados à saúde das mulheres na graduação, a grande maioria (85,2\%) relatou estar apta a realizar exame ginecológico para prevenção do câncer cervical, mas somente $47,8 \%$ faziam coleta de amostras para exame de Papanicolaou. Com relação ao exame das mamas para prevenção do câncer, 98,5\% informaram estar aptos a realizar o exame clínico, mas somente $59,6 \%$ o faziam. Muitos informaram que a coleta de citologia oncótica e o exame clínico das mamas eram realizados pela enfermeira, entretanto, não existem evidências científicas de boa qualidade demonstrando benefícios do exame clínico periódico das mamas para prevenção do câncer de mama. ${ }^{23}$

O cenário é estimulante quando se analisam as atividades de atenção pré-natal e puerperal: $87,6 \%$ dos médicos atendiam gestantes e $76,4 \%$ atendiam puérperas. Somente $22,1 \%$ relataram alguma dificuldade para atender gestantes e $34,5 \%$ para o atendimento puerperal. Entretanto, é escassa a prática de realizar o toque vaginal, pois 56,3\% informaram que não avaliam bacia materna durante o pré-natal. Por outro lado, somente 49,2\% dos médicos identificavam fatores de risco puerperais. A literatura evidencia que esses fatores de risco são infecçôes, hemorragias e síndromes hipertensivas, importantes causas de morbimortalidade materna no Brasil. ${ }^{20}$ Estes resultados mostram, potencialmente, a falta de um cuidado mais qualificado nesta fase do ciclo gestacional, pois muitas das complicaçôes puerperais exigem diagnóstico e conduta ágeis.

As dificuldades mais frequentes se relacionaram às atividades voltadas para a atenção no climatério e saúde reprodutiva. Apesar de 69,3\% informarem atender mulheres no climatério, somente $35,4 \%$ rastreavam as doenças previstas, enquanto apenas $27,5 \%$ solicitavam os exames para prevenir/diagnosticar essas doenças. A terapia hormonal (TH), entretanto, apresenta inúmeras controvérsias e deve se restringir a casos selecionados, pois existem importantes riscos quando não são respeitadas suas contraindicações. ${ }^{24,25} \mathrm{O}$ apoio matricial e a criação de espaços coletivos para discussôes e planejamento, previstos nas diretrizes do NASF (Núcleo de Apoio à Saúde da Família) podem auxiliar a resolver dificuldades relacionadas aos cuidados de saúde das mulheres climatéricas. ${ }^{9}$

Por sua vez, 75,6\% dos médicos prestavam cuidados à saúde reprodutiva (planejamento familiar e infertilidade), mas somente $14,8 \%$ solicitavam os exames adequados. ${ }^{9,19,20}$ As dificuldades mais importantes na atenção à saúde reprodutiva foram aquelas relacionadas à infertilidade, porém, com o apoio matricial (NASF), poder-se-ia ajudar a resolver as dificuldades relacionadas aos cuidados ao casal infértil. ${ }^{9}$

Evidenciou-se que os médicos náo se envolvem tanto com atividades educativas, em especial os mais jovens, por vários motivos: a) as atividades não ocorrem na sua unidade de saúde; b) a agenda de consultas é muito extensa; c) não têm interesse; d) as atividades são desenvolvidas pelos enfermeiros. Estudo analisando atividades das equipes da ESF de 10 municípios brasileiros com mais de 100 mil habitantes, também verificou baixa proporção de médicos realizando atividades de educação em saúde, variando de $22 \%$ a $53 \%{ }^{6}$

Finalmente, percebe-se que médicos formados há até três anos relataram mais dificuldades, suscitando as seguintes hipóteses: a) os médicos estão delegando funçôes para outros profissionais e deixando de realizar certos procedimentos; b) as DCN não estão sendo suficientemente efetivas para reorientar a formação médica, de forma a ter um profissional capaz de prestar cuidados à saúde. Deve-se também considerar que os médicos, quando alunos, podem ter se envolvido insuficientemente com atividades de Ginecologia e Obstetrícia, explicando, em parte, suas deficiências. Também, destaca-se a necessidade de inclusão da MFC como disciplina no curso de graduação de forma a propiciar aos estudantes vivenciarem e entenderem o cenário em que vivem as pessoas que eles irão atender, possibilitando-lhes desenvolver uma visão integral do cuidado à saúde. ${ }^{12}$

Vasconcelos e Zaniboni ${ }^{1}$ entrevistaram 54 médicos na ESF do município de São Paulo, verificando que 39,0\% tinham tempo menor que cinco anos de formação e que a principal motivação para trabalhar na ESF era o interesse em atuar na APS (aproximadamente 25\%). Não deram destaque à atenção à saúde da mulher e observaram que as principais dificuldades encontradas foram: alta demanda de consultas, elevada incidência de casos complexos, falta de incentivo à especializaçáo. Constataram também um déficit de médicos de família (20\%, em média) em 27 regiōes do município. Segundo os autores, esse déficit teria várias causas relacionadas à graduação: falta de vivência dos alunos na ESF; enfoque hospitalocêntrico das faculdades; desinteresse do aluno em atuar na ESF.

Para Ciuffo e Ribeiro, ${ }^{21}$ escolas médicas representam espaços privilegiados de produção de conhecimento e discussão, mas ainda reproduzem a lógica fragmentada do cuidado às pessoas, deixando de potencializar o que há de mais rico no campo da educação: as relações possíveis de serem desenvolvidas entre quem cuida e quem recebe o cuidado. Analisando as falas de professores e estudantes de dois cursos de Medicina, verificaram que os docentes percebiam a importância do compromisso social que a profissão exige, assim como a necessidade de mudanças no processo de formação dos médicos, apesar de vários deles serem resistentes a mudanças. Os estudantes, por sua vez, demonstraram descontentamento com a 
formação, apesar de reconhecerem existir indícios de ruptura com os tradicionais paradigmas da formação médica. Por fim, os autores discutem a necessidade da construção de um diálogo entre escolas médicas e o SUS, enfatizando o princípio da integralidade como eixo estruturante dessa formação.

Anderson et al. ${ }^{12}$ destacam a importância da participação dos profissionais da Medicina de Família e Comunidade nos cursos de graduação em Medicina, tendo em vista que seus preceitos e objetivos relacionam-se diretamente aos da APS. Segundo os autores, a MFC pode ter importante papel estratégico nas escolas médicas que implantaram reformas no ensino, para atender às necessidades de saúde da população.

Reforçando as sugestôes dos autores anteriores, foram recentemente publicadas as "Diretrizes para o ensino na Atenção Primária à Saúde na Graduação em Medicina” com o objetivo de apoiar escolas médicas na elaboração de projetos políticopedagógicos, reforçando a diversificação de cenários de prática. Destaca-se a necessidade de que o ensino na APS esteja presente longitudinalmente, ao longo de todo o curso, e que deva fazer parte do núcleo de ensino e da prática clínica dos futuros médicos. ${ }^{26}$

Estudo de Montekio et al., ${ }^{27}$ analisando o sistema de saúde público e privado brasileiro, constatou que o Brasil segue apresentando os piores indicadores de saúde entre os países de melhor renda da América Latina. Entre esses indicadores, o mais preocupante é a taxa de mortalidade materna, que se mantém elevada, apesar de a ESF oferecer cobertura universal a $50 \%$ da população brasileira. Outro indicador apresentado foi morte por câncer de mama, que ainda se mantém elevado entre as mulheres. Levantamos as seguintes questôes: se a maioria das gestantes e puérperas são atendidas na ESF e se é meta do SUS fazer prevenção do câncer de mama, por que as mortes pelas causas apontadas não estão diminuindo? Não teriam os profissionais de saúde as competências necessárias para prover os cuidados adequados e evitar as mortes passíveis de prevenção? A baixa resolubilidade do sistema seria responsável por essa baixa eficiência? Para responder a estas questôes, seria necessário que instâncias prestadoras de cuidados e formadoras de médicos estivessem sintonizadas e comprometidas. Desse modo poderíamos vislumbrar a melhoria dos indicadores de saúde das mulheres no Brasil.

Uma possível fragilidade do presente estudo é ter se baseado em questionários que foram respondidos por médicos voluntários. Pode-se imaginar um viés, pois foram os interessados que se dispuseram a responder às questóes. Por outro lado, a fortaleza do estudo reside no seu grande tamanho amostral e nas várias coincidências de respostas, sobretudo no que diz respeito às dificuldades encontradas para prover a assistência à saúde das mulheres.

\section{Conclusões}

Pode-se concluir que, em geral, os médicos com até três anos de formação relataram mais dificuldades em todos os tipos de cuidados à saúde das mulheres. Presume-se que as deficiências assinaladas pelos médicos mais jovens estejam relacionadas a falhas na sua formação, durante a graduação, associadas à mudança de práticas nas unidades de saúde - com delegação das açôes para outros profissionais de saúde - e ao reduzido tempo de exercício profissional que pudesse corrigir deficiências de formação. Essa hipótese necessita ser confirmada por outros estudos, mas é um alerta às escolas médicas do Brasil. Fica evidente a necessidade de prover ensino e campos de prática adequados, além da educação permanente dos profissionais, de forma a garantir que os futuros médicos adquiram as competências mínimas necessárias para prestarem cuidados de atenção à saúde das mulheres brasileiras.

\section{Referências}

1. Vasconcelos FGA, Zaniboni MRG. Dificuldades do trabalho médico no PSF. Ciênc Saúde Coletiva. 2011; 16(Supl. 1): 1494-1504. http://dx.doi. org/10.1590/S1413-81232011000700085

2. Falk JW, Gusso G, Lopes JMC. Medicina de Família e Comunidade com Especialidade Médica e Profissão. In: Gusso G, Lopes JMC. Tratado de Medicina de Família e Comunidade: princípios, formação e prática. Porto Alegre: Artmed; 2012. p. 12-16.

3. World Health Organization (WHO). Relatório da Conferência Internacional sobre Cuidados de Saúde Primários (Internet). Declaração de Alma-Ata: 1978. [online] [acesso em 2012]. Disponível em: http://www.saudepublica.web.pt/05-promocaosaude/Dec_Alma-Ata.htm 
4. Rodrigues RN, Anderson MIP. Saúde da Família: uma estratégia necessária. Rev Bras Med Fam Comunidade. 2011; 6(18): 21-24. http://dx.doi. org/10.5327/Z1809-59092011001800006

5. Sampaio LFR, Mendonça CS, Lermen N Jr. Atenção Primária à Saúde no Brasil. In: Gusso G, Lopes JMC. Tratado de Medicina de Família e Comunidade: princípios, formação e prática. Porto Alegre: Artmed; 2012. p. 28-41.

6. Escorel S, Giovanella L, Mendonça MHM, Senna MCM. O Programa de Saúde da Família e a construção de um novo modelo para a atenção básica no Brasil. Rev Panam Salud Publica. 2007; 21(2): 164-176. http://dx.doi.org/10.1590/S1020-49892007000200011

7. Secretaria de Estado de Saúde (MG). Deliberação CIB-SUS/MG n 180, 26 de julho de 2005. [on line] [acesso 2012]. Minas Gerais; 2005. Disponível em: http://www.saude.mg.gov.br/atos_normativos/deliberacoes/2005/del_180.pdf

8. Minas Gerais. Saúde. Cumprimento antecipado dos Objetivos do Milênio. [Online] [acesso em 2013]. Disponível em: http://www.mg.gov.br/ governomg/portal/c/governomg/conheca-minas/minas-em-numeros/12435-saude/517077-resultados-do-governo-de-minas-na-gestao-antonioanastasia-saude/5146/5044

9. Ministério da Saúde (BR). Diretrizes do NASF: Núcleo de Apoio Saúde da Família. Brasília: Ministério da Saúde; 2010 . (Cadernos de Atenção Básica, n. 27) (Série A. Normas e Manuais Técnicos). Disponível em: http://189.28.128.100/dab/docs/publicacoes/cadernos_ab/abcad27.pdf

10. Harris M, Haines A. Brazil's Family Health Programme. Editorial. BMJ 2010; 341:c4945. PMid:21115587. http://dx.doi.org/10.1136/bmj.c4945

11. Mendonça MHM, Martins MIC, Giovanella L, Escorel S. Desafios para gestão do trabalho a partir de experiências exitosas de expansão da Estratégia de Saúde da Família. Ciênc Saúde Coletiva. 2010; 15(5): 2355-2365. http://dx.doi.org/10.1590/S1413-81232010000500011

12. Anderson MIP, Demarzo MMP, Rodrigues RD. A Medicina de Família e Comunidade, a Atenção Primária à Saúde e o Ensino de Graduação: recomendações e potencialidades. Rev Bras Med Fam Comunidade. 2007; 3(11): 157-72.

13. Campos CVA, Malik AM. Satisfação no trabalho e rotatividade dos médicos do Programa de Saúde da Família. RAP. 2008; 42(2): 347-68.

14. Ministério da Saúde (BR). Saúde da Família: avaliação da implementação em dez grandes centros urbanos: síntese dos principais resultados. 2. ed. atual. Brasília: Ministério da Saúde, 2005. (Série C. Projetos, Programas e Relatórios). Disponível em: http://189.28.128.100/dab/docs/publicacoes/ geral/miolo_saude_familia.pdf

15. Ronzani TM, Van Stralen CJ. Dificuldades de Implantação do Programa de Saúde da Família como Estratégia de Reforma do Sistema de Saúde Brasileiro. Rev APS. 2003; 6(2): 99-107. Disponível em: http://www.ufff.br/nates/files/2009/12/Gerencia2.pdf

16. Anderson MIP, Gusso G, Castro ED F. Medicina de Família e Comunidade: especialistas em integralidade. Rev APS. 2005; 8(1): 61-67. Disponível em: http://www.ufjf.br/nates/files/2009/12/FamiliaComun.pdf

17. Ministério da Saúde (BR). Secretaria de Gestão do Trabalho e da Educação na Saúde. Programa Nacional de Reorientação da Formação Profissional em Saúde (Pró-Saúde). [on line] [acesso em 2012]. Disponível em: http://www.prosaude.org/

18. Silvério JB. Programa de educação permanente para médicos de família. Rev Med Minas Gerais. 2008; 18(Supl 4): S60-S66. Disponível em: http:// rmmg.medicina.ufmg.br/index.php/rmmg/article/viewArticle/104

19. Ministério da Saúde (BR). Rastreamento. Brasília: Ministério da Saúde, 2010. (Série A. Normas e Manuais Técnicos) (Cadernos de Atenção Primária n. 29). Disponível em: http://189.28.128.100/dab/docs/publicacoes/cadernos_ab/abcad29.pdf

20. Secretaria de Estado de Saúde (MG). Atenção ao pré-natal, parto e puerpério: protocolo Viva Vida. 2 ed. Belo Horizonte: SAS/SES, 2006.84 p. [Online] [acesso em 2013]. Disponível em: https://www.nescon.medicina.ufmg.br/biblioteca/imagem/2016.pdf

21. Ciuffo RS, Ribeiro VMB. Sistema Único de Saúde e a formação dos médicos: um diálogo possível? Interface Comunicação Saúde Educação. 2008; 12(24): 125-40. http://dx.doi.org/10.1590/S1414-32832008000100010

22. Ministério da Educação (BR). Conselho Nacional de Educação, Câmara de Educação Superior. Diretrizes Curriculares Nacionais do Curso de Graduação em Medicina. Homologada em 03/10/2001, Parecer CES 1.133/2001. Resolução CNE/CES nº 4, de 7/11/2001. Brasilia: Ministério da Educação; 2001. [on line] [acesso em 2011]. Disponível em: http://portal.mec.gov.br/cne/arquivos/pdf/CES04.pdf

23. Canadian Task Force on Preventive Health Care. Screening for Breast Cancer. Clinical Breast Exam. [online] [acesso em 2013]. Disponível em: http:// canadiantaskforce.ca/guidelines/2011-breast-cancer/

24. Federação Brasileira das Associações de Ginecologia e Obstetrícia (FEBRASGO). Manual de Orientação - Climatério. São Paulo: FEBRASGO; 2010 [acesso em 2013 Abr 13]. Disponível em: http:// www.febrasco.org.br

25. Sociedade Brasileira de Cardiologia. Associação Brasileira do Climatério. I Diretriz brasileira sobre prevenção de doenças cardiovasculares em mulheres climatéricas e a influência da terapia de reposição hormonal (TRH). Arq Bras Cardiol. 2008; 91(1 supl.1): 1-23. Disponível em: http://www. ipgs.com.br/cursos/documents/2008_Diretriz_Prevenc\%E3o_Doencas_Cardiovasculares_Mulheres_Climatericas.pdf

26. Demarzo MMP, Almeida RCC, Marins JJN, Trindade TG, Anderson MIP, Stein AT et al. Diretrizes para o ensino na Atenção Primária à Saúde na graduação em Medicina. Rev Bras Med Fam Comunidade. 2011; 6(19): 145-50. 20-22. http://dx.doi.org/10.5712/rbmfc6(19)116

27. Montekio VB, Medina G, Aquino R. Sistema de salud de Brasil. Salud Pública México. 2011; 53(Supl 2): S120-S131. 21-23. Disponível em: http:// www.scielo.org.mx/scielo.php?script=sci_arttext\&pid=S0036-36342011000800008\&lng=es. 